\title{
Estado nutricional de los pacientes con diagnóstico de Niemann-Pick tipo C en Colombia
}

\author{
Nutritional Status of Patients with Niemann-Pick type C \\ diagnosis in Colombia
}

\author{
Liliana Ladino Meléndez ${ }^{1 *}$, Ana Lorena Montealegre Páez', Erika Ochoa ${ }^{2}$ \\ Recibido: 2 de febrero de 2019. Aceptado para publicación: 11 de marzo de 2019 \\ Publicado en línea, marzo 23 de 2019 \\ https://doi.org/10.35454/rncm.v2n1.062
}

\section{Resumen}

Introducción: la enfermedad de Niemann Pick tipo C, es una enfermedad neurovisceral de depósito lisosomal progresiva y debilitante cuyos síntomas tienen un impacto negativo en el estado nutricional de las personas que lo padecen. Sumado a lo anterior, se han descrito complicaciones gastrointestinales severas con el uso de Miglustat, tratamiento de elección para esta enfermedad, lo cual podría incrementar el riesgo nutricional de estos pacientes.

Objetivo: describir el estado nutricional antropométrico y la ingesta dietética de 15 pacientes colombianos con NiemannPick tipo C.

Métodos: previo consentimiento informado, se realizó valoración nutricional en donde se obtuvieron datos demográficos, antropométricos y de ingesta dietética de los pacientes, los cuales fueron digitados en una base de datos elaborada en Excel donde se calcularon los siguiente indicadores: peso para la edad, peso para la longitud-talla y longitud-talla para la edad de Waterlow, índice de masa corporal para la edad, perímetro del brazo para la edad y pliegue cutáneo del tríceps para la edad, para el análisis de la información se empleó estadística descriptiva.

Resultados: se encontró que todos los pacientes presentan alteración en el estado nutricional antropométrico, y solo la mitad de los pacientes cubren los requerimientos energéticos.

Conclusión: existe la necesidad de realizar nuevos estudios que identifiquen la relación entre el tratamiento, y las alteraciones nutricionales y gastrointestinales de los pacientes y cómo esto puede afectar el pronóstico y la sobrevida de los pacientes.

Palabras clave: errores innatos del metabolismo, enfermedades de depósito lisosomal, estado nutricional, crecimiento y desarrollo.

1. Instituto de Investigación en Nutrición, Genética y Metabolismo. Facultad de Medicina. Universidad El Bosque, Bogotá, D.C., Colombia.

\section{Summary}

Introduction: Niemann Pick type C disease is a neurovisceral disease of progressive and debilitating lysosomal deposit whose symptoms have a negative impact on the nutritional status of people who suffer from it. In addition to the above, severe gastrointestinal complications have been described with the use of Miglustat, the treatment of choice for this disease, which could increase the nutritional risk of these patients.

Objective: To describe the anthropometric nutritional status and dietary intake of 15 Colombian patients with Niemann-Pick type $C$ disease.

Methods: After informed consent, nutritional assessment was carried out, where demographic data, anthropometric data and dietary intake data of the patients was obtained. The data obtained was entered in a database prepared in Excel where the following indicators were calculated: weight for age, weight for length and length-height for age of Waterlow, body mass index for age, perimeter of the arm for age and triceps skin fold for age, descriptive statistics were used to analyze the information.

Results: It was found that all patients presented alteration in the anthropometric nutritional status and only half of the patients cover the energy requirements.

Conclusion: There is a need to carry out new studies that identify the relationship between treatment and nutritional and gastrointestinal alterations of patients and how this can affect the prognosis and survival of patients.

Keywords: Metabolism inborn errors; Lysosomal Storage Diseases; Nutritional status; Growth and development.

2. Instituto Tecnológico y de Estudios Superiores de Monterrey. ITESM Campus Ciudad de México, México.

*Correspondencia: Liliana Ladino Meléndez.

lladinom@unbosque.edu.co 


\section{INTRODUCCIÓN}

Las enfermedades de depósito lisosomal hacen parte de un grupo de enfermedades denominadas errores innatos del metabolismo y se caracterizan no solo por ser progresivas sino por además manifestarse durante la niñez ${ }^{(1,2)}$. Se estima que dentro de este tipo de enfermedades huérfanas existen alrededor de 50 condiciones patológicas ${ }^{(2)} y$ aunque su frecuencia suele ser baja, entre todas afectan a una parte significativa de la población $^{(2-4)}$. Una de ellas es la enfermedad de NiemannPick Tipo C (NP-C), descrita por primera vez en 1920 gracias a los trabajos realizados por Albert Niemann y Ludwing Pick ${ }^{(5)}$. Es una enfermedad de herencia autosómica recesiva y se caracteriza por el depósito lisosomal de esfingomielina debido al déficit de la enzima esfingomielinasa, la cual degrada esfingolípidos. El acúmulo de estas sustancias ocasiona lisis celular en cerebro, hígado y bazo ${ }^{(5-13)}$. Por lo tanto, aunque las manifestaciones clínicas suelen ser heterogéneas ${ }^{(14)}$ es usual que estén presentes en los órganos donde se produce la acumulación de esfingolípidos y colesterol, lo que la convierte en una condición neurovisceral ${ }^{(5,6,12)}$. Además, los pacientes están en un continuo riesgo nutricional debido a los diferentes síntomas que se presentan, como la pérdida de tono y coordinación muscular lo cual suele dificultar la ingesta de alimentos, y la afectación cerebral ${ }^{(15-18)}$.

Por su parte, el tratamiento usual de los pacientes con NP-C es la terapia de inhibición de sustrato Miglustat ${ }^{\oplus}{ }^{(19-32)}$ la cual está aprobada por la Food and Drug Administration (FDA) desde el año $2003^{(33,34)}$ y en Colombia desde $2009^{(35)}$. Sin embargo, esta terapia trae consigo complicaciones gastrointestinales, debido a la inhibición de las disacaridasas intestinales, lo cual ocasiona una inadecuada hidrólisis de carbohidratos y la subsecuente diarrea osmótica con alteración en la fermentación colónica, que posteriormente producen pérdida de peso ${ }^{(25,36-38)}$. Debido a las complicaciones gastrointestinales ocasionadas por la terapia de inhibición de sustrato y como parte de un tratamiento integral, el mantener un adecuado manejo nutricional puede minimizar las complicaciones, evitando la pérdida de peso que pueda ocasionar desnutrición, mejorando la calidad de vida del paciente y su familia ${ }^{(36,39)}$. El objetivo de la presente investigación fue describir el estado nutricional antropométrico y la ingesta dietética de 15 pacientes colombianos con Niemann-Pick tipo C quienes reciben terapia con Miglustat ${ }^{\circledR}$.

\section{MÉTODOS Y MATERIALES}

Antes de iniciar la consulta, a cada paciente se solicitó el consentimiento y asentimiento informado, en donde se garantizaba la confidencialidad de los datos y la información obtenida. A los 15 pacientes diagnosticados con NP-C, se les realizó valoración nutricional en la cual se registraron los siguientes datos: sexo, fecha de nacimiento, edad, grupo etario, tipo y lugar de vivienda, nivel socioeconómico familiar, edad de diagnóstico y edad de inicio de la terapia de inhibición de sustrato Miglustat ${ }^{\circledast}$.

La valoración nutricional antropométrica incluyó: peso $(\mathrm{P})$, longitud $(\mathrm{L})$ o talla $(\mathrm{T})$, perímetro cefálico $(\mathrm{PC})$, perímetro del brazo (PB), pliegue cutáneo del tríceps (PCT). Las mediciones fueron realizadas por una nutricionista dietista certificada en ISAK 1. Para determinar el peso se empleó una balanza digital Tanita referencia UM-061 Body fat/body water, la longitud se determinó con infantómetro marca SECA 210, la talla fue tomada con tallímetro portátil marca SECA 213. Los perímetros fueron medidos con cinta métrica marca SECA 201 y el PCT fue tomado con adipómetro Slim Guide.

Los datos recolectados fueron digitados en una base de datos elaborada en Excel y partiendo de los datos antropométricos se calcularon los indicadores: peso para la edad (P/E) de Gómez ${ }^{(40)}$, peso para la longitud-talla $(\mathrm{P} / \mathrm{L}-\mathrm{T})$ y longitud-talla para la edad (L-T/E) de Waterlow ${ }^{(41)}$, índice de masa corporal para la edad (IMC/E), perímetro del brazo para la edad $(\mathrm{PB} / \mathrm{E})$ y pliegue cutáneo del tríceps para la edad (PCT/E), empleando los estándares de crecimiento de la Organización Mundial de la Salud (OMS) ${ }^{(42)}$. Para evaluar los indicadores antes mencionados en los menores de 18 años, se utilizaron los puntos de corte de la Resolución 2121 del 9 de junio de 2010 $0^{(43)}$ la cual estaba vigente para la fecha del desarrollo del proyecto y a su vez, se utilizaron los puntos de corte de la OMS para los mayores de 18 años junto con los de la Sociedad Española para el Estudio de la Obesidad $(\text { SEEDO })^{(44)}$. También se estimaron los porcentajes de reservas corporales de masa magra (RMM) y grasa (RMG) los cuales fueron evaluados con los puntos de corte de Frisancho ${ }^{(45)}$ para todas las edades.

La recolección de datos de ingesta dietética se realizó por medio de recordatorio de 24 horas de tres días ( 2 días entre semana y 1 día de fin de semana), donde se indagaron todos los alimentos ingeridos durante el día, permitiendo conocer las preparaciones ingeridas y cada uno de los ingredientes contenidos. Partiendo 
de la información recolectada, se estimó el aporte de energía basado en el análisis nutricional de cada uno de los alimentos ${ }^{(46)}$ y se reportó el aporte energético total (Kilocalorías día y Kilocalorías por Kilogramo día) aporte de macronutrientes y micronutrientes (dados en unidades por Kilogramo por día). Se empleó estadística descriptiva para el análisis de la información.

\section{RESULTADOS}

Uno de los síntomas más preocupantes de los pacientes con NP-C es la disfagia, la cual con el tiempo tiende a empeorar y en muchos de los casos se requieren medidas externas o cambios en la consistencia de la comida para mantener una nutrición adecuada ${ }^{(9,47,48)}$. En nuestra serie de casos la población de niños fue de 86,7 \% ( $\mathrm{n}=13)$; la evaluación antropométrica de los 13 niños demostró que seis $(46,2 \%)$ sufrían desmedro, dos de estos también cursaban con emaciación; dos padecían emaciación $(15,4 \%)$ y únicamente cinco pacientes tenían estado antropométrico normal. Respecto al estado nutricional de la población adulta, los dos pacientes se encontraban en normalidad según el IMC y la clasificación de la OMS, no obstante, uno de ellos tenía talla de $137 \mathrm{~cm}$, $10 \mathrm{~cm}$ por debajo del promedio en la población femenina colombiana. La composición corporal resultó heterogénea siendo que $60 \%$ de los pacientes presentaron algún grado de depleción de masa magra (estimada por área muscular de brazo) mientras que la reserva de masa grasa (estimada por pliegue cutáneo tricipital) se encontró disminuida en 10 pacientes, tres con exceso y dos en normalidad. Los indicadores antropométricos y la composición corporal de los pacientes se pueden observar en la Tabla 1, cabe resaltar que no se evidencia diferencia estadísticamente significativa en la composición corporal según el sexo.
Con relación a la evaluación dietética de energía, la dieta promedio aportaba 73,8 $\pm 45,3 \mathrm{kcal} / \mathrm{kg} /$ día, el aporte proteico fue en promedio $3,3 \pm 1,9 \mathrm{~g} / \mathrm{kg} /$ día de proteína sin existir diferencias entre género; el aporte de carbohidratos correspondió a 7,5 $\pm 5,0 \mathrm{~g} / \mathrm{kg} /$ día y respecto a la grasa a $60,1 \pm 21,2 \mathrm{~g} / \mathrm{kg} /$ día. En la Tabla 2 se reportan características de la ingesta dietaria de energía y proteína de los pacientes del presente estudio. Es imperativo resaltar que ni el aporte adecuado, excesivo, ni deficiente, se relacionó con un estado nutricional en particular, demostrando la necesidad de evaluar el requerimiento energético de estos pacientes por calorimetría indirecta o agua doblemente marcada.

\section{DISCUSIÓN}

La NP-C se encuentra en la actualidad dentro de la lista de enfermedades huérfanas del Ministerio de Salud y Protección Social de nuestro país (Resolución 2048 de $2015)^{(49)}$, hoy en día es difícil encontrar cifras sobre su prevalencia en Colombia, por eso estos datos son de vital importancia al reportar la totalidad de pacientes con NP-C en tratamiento con Miglustat ${ }^{\oplus}$ al cierre del estudio durante el año 2015. Sin embargo, la mayoría de los datos existentes suelen ser cifras mundiales o europeas $^{(50)}$. Como sucede con muchas de estas enfermedades, los pacientes suelen ser subdiagnósticados por diferentes motivos, ya sea la dificultad en el acceso a los servicios de salud o el desconocimiento de los profesionales de la salud sobre estas condiciones ${ }^{(3)}$, lo que no solo dificulta la recolección de datos epidemiológicos confiables, sino que además pone en riesgo la vida de estos pacientes, quienes muchas veces presentan necesidades nutricionales específicas y obligatorias.

Alrededor del mundo, son pocos los estudios que se refieren al estado nutricional de los pacientes con diag-

Tabla 1. Indicadores antropométricos y composición corporal

\begin{tabular}{|c|c|c|c|c|c|c|c|c|}
\hline Sexo & $\begin{array}{c}\text { P/E } \\
\text { z-score } \\
(\mathbf{n}=\mathbf{8})\end{array}$ & $\begin{array}{c}\text { P/T } \\
\text { z-score } \\
(n=9)\end{array}$ & $\begin{array}{c}\text { T/E } \\
\text { z-score } \\
(n=13)\end{array}$ & $\begin{array}{l}\text { IMC/E } \\
\text { z-score } \\
(n=13)\end{array}$ & $\begin{array}{c}\text { IMC } \\
\mathrm{mg} / \mathrm{m}^{2} \\
(\mathrm{n}=2)\end{array}$ & $\begin{array}{c}\text { PB } \\
\text { cm } \\
(n=15)\end{array}$ & $\begin{array}{l}\text { Área muscular de brazo } \\
\qquad \begin{array}{c}\text { mm } \\
(n=15)\end{array}\end{array}$ & $\begin{array}{l}\text { PCT } \\
\text { mm } \\
(n=15)\end{array}$ \\
\hline Masculino & $1,5(1,8)$ & $-0,6(1,1)$ & $-0,8(1,7)$ & $-1,2(2,3)$ & \multirow[t]{2}{*}{$23,5(1,6)$} & $16,5(6,5)$ & $1635,1(1866,5)$ & $7(2)$ \\
\hline Femenino & $-2,2(2)$ & $-1,6(1,9)$ & $-2,4(0,1)$ & $-1,4(5,1)$ & & $17,65(8,2)$ & $1651,8(1725,3)$ & $8,5(5)$ \\
\hline Total & $-1,7(2,1)$ & $-1,4(1,5)$ & $-1,4(1,9)$ & $-1,4(2,5)$ & $23,5(1,6)$ & $17,4(7,3)$ & $1636,1(1958,5)$ & $7(6)$ \\
\hline $\begin{array}{l}\text { Diferencia entre } \\
\text { sexos }(p)\end{array}$ & 0,4561 & 0,6242 & 0,1871 & 0,7694 & NA & 0,5956 & 0,4795 & 0,1900 \\
\hline
\end{tabular}

Valores expresados en mediana (Rango Intercuartil) - Test de Mann-Whitney para diferencias entre sexos. 
Tabla 2. Aporte energético y proteico

\begin{tabular}{|c|l|c|c|c|c|}
\hline \multirow{2}{*}{$\begin{array}{c}\text { Variables } \\
\text { categóricas } \\
\text { Energía (kcal/ } \\
\text { kg/día) }\end{array}$} & \multicolumn{4}{|c|}{ Aporte dietético } \\
\cline { 3 - 4 } & p-value & $\begin{array}{c}\text { Proteína } \\
\text { (g/día) }\end{array}$ & p-value & \\
\hline \multirow{2}{*}{ Sexo } & Masculino & $71(85,1)$ & 0,5956 & $3,1(3,3)$ & \multirow{2}{*}{0,5557} \\
\cline { 2 - 3 } & Femenino & $77,1(86,4)$ & & $2,6(2,4)$ & \\
\cline { 2 - 3 } & Total & $71(85,7)$ & & $3,1(3,4)$ & \\
\hline
\end{tabular}

Prueba de Mann-Whitney para comparar dos medianas (sexo); * $\mathrm{p}<0,05$

nóstico de NP-C ${ }^{(36)}$ y en nuestro país hasta el momento no existe ninguno, siendo esta la primera publicación que describe el estado nutricional de pacientes colombianos con este diagnóstico y que evidencia que es una población, que debido a su condición patológica presentan un riesgo nutricional. Aunque una de las limitaciones de este trabajo fue la no evaluación de síntomas gastrointestinales, ni la relación del tratamiento sobre el estado nutricional de los pacientes, se encontró que la prevalencia de deterioro en los 15 pacientes fue superior a la nacional en el momento del estudio (13,2\% para población de 0 a 5 años, $9 \%$ para población de 5 a 9 años y $10,7 \%$ para adolescentes de 10 a 17 años) $)^{(51)}$. De igual forma, la prevalencia de emaciación encontrada en los pacientes, es superior a la prevalencia reportada en el ámbito nacional (2,1\% para niños de 5 a 17 años). Estos datos demuestran que los pacientes con NP-C se encuentran en alto riesgo nutricional, siendo la talla lo que más se encuentra afectado.

Por todo lo anterior, se considera necesario identificar en futuros estudios nacionales o internacionales si los pacientes con NP-C están recibiendo un cuidado nutricional integral adecuado que incluya la valoración y seguimiento nutricional y a su vez, identificar el impacto real del tratamiento con Miglustat ${ }^{\circledR}$ sobre el estado nutricional de estos pacientes. Esto podría dar una mejor perspectiva a los profesionales de la salud sobre la forma de abordar este tipo de enfermedades y a los pacientes herramientas para que se empoderen de su salud y tomen las decisiones adecuadas en su propio beneficio ${ }^{(52)}$.

\section{CONCLUSIONES}

Todos los pacientes colombianos con NP-C presentan alteración en el estado nutricional antropométrico y solo la mitad de ellos cubren sus requerimientos ener- géticos; sin embargo, hacen falta estudios que identifiquen la relación entre el tratamiento y las alteraciones nutricionales y gastrointestinales de los pacientes, y cómo esto puede afectar su pronóstico y sobrevida; no solo de la enfermedad NP-C y sino también de otras enfermedades huérfanas.

\section{Agradecimientos}

Queremos agradecer a la organización Caminando Juntos por ayudar al reclutamiento de los pacientes. A Natalia Sepúlveda, N.D., MSc., docente de la Pontificia Universidad Javeriana por su contribución con la atención de pacientes del proyecto.

\section{Declaración de autoría}

Los autores declaran que han contribuido por igual en la concepción, realización de la investigación y en la redacción y revisión de este artículo.

\section{Financiación}

El presente estudio no tuvo financiación.

\section{Conflicto de intereses}

Los autores declaran no tener conflicto de intereses.

\section{Referencias bibliográficas}

1. Ruiz M, Sánchez F, Dalmau J, Gómez L. Tratamiento nutricional de los errores innatos del metabolismo. 2 da edición. Madrid, España: 2007. p. 4-32.

2. Márquez W, Linares A. Enfermedades de depósito lisosomal. CCAP. Sociedad Colombiana de Pediatría [Internet]. [citado el 3 de abril de 2018]. Disponible en: https://scp.com.co/ precop-old/precop_files/modulo_4_vin_3/deposito_lisosomal.pdf

3. Rosselli D, Rueda J. Enfermedades raras, huérfanas y olvidadas [Internet]. 2011. [citado el 3 de abril de 2018]. Disponible en:http://www.academia.edu/5271337/ESTUDIO_ ENFERMEDADES_RARAS_HUÉRFANAS_Y _ OLVIDADAS

4. García Díaz JD, Mesa Latorre JM, Corps Fernández D, Valbuena Parra. Enfermedades por depósito lisosomal. Medicine. 2016;12(19): 1072-81.

5. Vanier TM. Niemann-Pick disease type C. Orphanet J Rare Dis. 2010; 5:16.

6. Villamandos García D, Santos-Lozano A. Enfermedad de Niemann-Pick: un enfoque global. Ene [Internet]. 2014 [citado el 3 de abril de 2018];8(2). Disponible en: http:// 
scielo.isciii.es/scielo.php?script=sci_arttext\&pid=S1988348X2014000200003

7. Jean-Tron G, Ortega-Ponce F, Isalas-García D. Enfermedad de Niemann-Pick tipo C. Rev Mex Neuroci. 2012;13(5):281-5.

8. Platt FM, Boland B, Aarnoud C, van der S. Lysosomal storage disorders: The cellular impact of lysosomal dysfunction. J Cell Biol. 2012; 199(5):723-34.

9. National Organization for Rare Disorders (NORD). Niemann Pick Disease Type C [Internet]. Lugar y año desconocidos [citado el 4 de abril de 2018]. Disponible en https://rarediseases.org/rare-diseases/niemann-pick-disease-type-c/

10. U.S. National Library of Medicine. Genetics Home Reference. Niemann-Pick disease [Internet]. Estados Unidos; 2015 [citado el 2 de abril de 2018]. Disponible en https://ghr.nlm. nih.gov/condition/niemann-pick-disease\#

11. Sévin M, Lesca G, Baumann N, Millat G, Lyon-Caen O, Vanier MT, et al. The adult form of Niemann-Pick disease type C. Brain. 2007; 130(Pt1): 120-33.

12. Vanier MT. Niemann-Pick diseases. Handb Clin Neurol. 2013; 113: 1717-21.

13. Xiong H, Bao X, Zhang Y, Xu Y, Qin J, Shi H, et al. NiemannPick disease type C: analysis of 7 patients in World J Pediatr 2012;8(1):61-6.

14. Imrie J, Heptinstall L, Knight S, Strong K. Observational cohort study of the natural history of Niemann-Pick disease type $\mathrm{C}$ in the UK: a 5-year update from the UK clinical database. BMC Neurol. 2015;15:257.

15. U.S. Department of Health \& Human Services. National Center for Advancing Translational Sciences. Niemann- Pick disease [Internet]. Estados Unidos: Gaithersburg; 2017 [citado el 3 de abril de 2018]. Disponible en https://rarediseases.info.nih.gov/diseases/13334/niemann-pick-disease

16. Evans WR, Hendriksz CJ. Niemann-Pick type C disease - the tip of the iceberg? A review of neuropsychiatric presentation, diagnosis and treatment. BJPsych Bull. 2017; 41(2): 109-14.

17. Ulatowski L, Parker R, Davidson C, Yanjanin N, Kelley TJ, Corey D, et al. Altered vitamin E status in Niemann-Pick type C disease. J Lipid Res. 2011; 52(7): 1400-10.

18. Iturriaga C, Pineda M, Fernandez-Valero EM, Vanier MT, Coll MJ. Niemann-Pick C disease in Spain:Clinical spectrum and development of a disability scale. J Neurol Sci. 2006;249(1):1-6.

19. Pineda M, Weaith JE, Mengel E, Sedel F, Hwu WL, Rohrbacj $\mathrm{M}$, et al. Miglustat in patients with Niemann-Pick disease Type C (NP-C): A multicenter observational retrospective cohort study. Mol Genet Metab. 2009; 98(3):243-9.

20. Wraith JE, Imrie J. New therapies in the management of Niemann-Pick type C disease: clinical utility of Miglustat. The Clin Risk Manag. 2009;5: 877-87.

21. Héron B, Valayannopoulos V, Baruteau J, Chabrol B, Ogier H, Latour P, et al. Miglustat therapy in the French cohort of paediatric patients with Niemann-Pick disease type C. Orphanet J Rare Dis. 2012; 7:36.
22. Wraith JE, Vecchio D, Jacklin E, Abel L, Chadha-Boreham $\mathrm{H}$, Luzy C, et al. Miglustat in adult and juvenile patients with Niemann-Pick disease type C: Long-term data from a clinical trial. Mol Genet Metab. 2010; 99(4):351-7.

23. Lachmann RH, Platt FM. Substrate reduction therapy for glycosphingolipid storage disorders. Expert Opin Investig Drugs. 2001; 20(3): 455-66.

24. Rosenbaum AI, Maxfield FR. Niemann-Pick type C disease: molecular mechanisms and potential therapeutic approaches. J Neurochem. 2011; 116(5):789-95.

25. Lyseng-Williamson KA. Miglustat: A Review of Its Use in Niemann-Pick Disease Type C. Drugs. 2014; 74(1): 61-74.

26. Santos-Lozano A, Villamandos García D, Sanchis-Gomar F, Fiuza-Luces C, Pareja-Galeano H, Garatachea N, et al. Niemann-Pick disease treatment: a systematic review of clinical trials. Ann Transl Med. 2015; 3(22):360.

27. U.S. National Library of Medicine Clinical Trials. gov [Internet]. Study of IV VTS-270 for Infantile Liver Disease Associated with Niemann-Pick Disease, Type C. Estados Unidos: Missouri; 2018. [citado el 2 de abril de 2018]. Disponible en: https://clinicaltrials.gov/ct2/show/record/ NCT03471143?term=Niemann-Pick+Disease\&rank=3

28. Patterson MC, Hendriksz CJ, Walterfang M, Sedel F, Vanier $\mathrm{MT}$, et al. Recommendations for the diagnosis and management of Niemann-Pick disease type C: An update. Mol Genet Metab. 2012; 106(3):330-44.

29. Patterson MC, Mengel E, Vanier MT, Schwierin B, Muller A, Cornelisse A, et al. Stable or improved neurological manifestations during miglustat therapy in patients from the international disease registry for Niemann-Pick disease type C: an observational cohort study. Oprhanet J Rare Dis. 2015;10:65.

30. Patterson MC, Vecchio D, Jacklin E, Abel L, Chadha-Boreham $\mathrm{H}$, Luzy C, et al. Long-Term Miglustat Therapy in Children With Niemann-Pick Disease Type C. J Child Neurol. 2010; 25(3):300-5.

31. Pineda M, Perez-Poyato MS, O'Callaghan M, Villaseca MA, Pocovi M, Domingo R, et al. Clinical experience with miglustat therapy in pediatric patients with Niemann-Pick disease type C: A case series. Mol Genet Metab. 2010; 99(4): 358-66.

32. Papandreou A, Gissen P. Diagnostic workup and management of patients with suspected Niemann-Pick type C disease. The Adv Neurol Disord. 2016; 9(3): 216-29.

33. Estados Unidos. Food and Drug Administration. Withholds approval of Zabesca as treatment for Niemann-Pick disease type C. Fort Atkinson: Wisconsin. 2010.

34. accessdata.fda.gov [Internet]. Silver Spring: U.S. Food and Drug Administration Drug; 2002 [citado el 4 de abril de 2018]. Disponible en: https://www.accessdata.fda.gov/ drugsatfda_docs/nda/2003/21-348_Zavesca.cfm

35. Colombia. Instituto Nacional de Vigilancia de Medicamentos y Alimentos-INVIMA. Sala especializada de medicamentos y productos biológicos de la comisión revisora. Acta No 27 . Bogotá: Ministerio de la Protección Social. 2009. 
36. Belmatoug N, Burlina A, Giraldo P, Hendriksz CJ, Kuter DJ, Mengel E, et al. Gastrointestinal disturbances and their management in miglustat-treated patients. J Inherit Metab Dis. 2011; 34(5):991-1001.

37. Amiri M, Naim HY. Miglustat-induced intestinal carbohydrate malabsorption is due to the inhibition of $\alpha$-glucosidases, but not $\beta$-galactosidases. J Inherit Metab Dis. 2012; 35:949-54.

38. Amiri M, Naim HY. Long term differential consequences of miglustat therapy on intestinal disaccharidases. J Inherit Metab Dis. 2014;37(6):929-37.

39. Champion H, Ramaswami U, Imrie J, Lachmann RH, Gallagher J, Cox TM, et al. Dietary modifications in patients receiving miglustat. J Inherit Metab Dis. 2010; 33(3):S379-83.

40. Gómez F. Desnutrición. Bol Med Hosp Infant Mex. 1991;54:299-304.

41. Waterlow. Classification and definition of protein-calorie malnutrition. Br Med J. 1972; 3(5826):566-9.

42. Onis M, Onyango A, Borghi E, Siyam A, Nishida C, Siekmann J. Development of a WHO growth reference for school-aged children and adolescents. Bull World Health Organ. 2007; 85 (9):660-7.

43. Colombia. Ministerio de la Protección Social. Resolución número 2121 de 2010. Bogota: 2010.

44. Sociedad Española para el estudio de la Obesidad (SEEDO). Consenso SEEDO' 2000 para la evaluación del sobrepeso y la obesidad y el establecimiento de criterios de intervención terapéutica. Med Clin (Barc). 2000;115:587-97.
45. Frisancho AR. Triceps skin Fol. And upper arm muscle size normal for assessment of nutricional status. Am J Clin Nutr. 1974;27:1052-8.

46. Instituto Colombiano de Bienestar Familiar. Tabla de Composición de Alimentos Colombianos (TCAC) [Internet]. [citado el 2 de abril de 2018]. Disponible en: https://www.icbf. gov.co/sites/default/files/tcac_2015_final_para_imprimir.pdf

47. Abela L, Plecko B, Palla A, Burda P, Nuoffer JM, Ballhausen D, et al. Early co-occurrence of a neurologic-psychiatric disease pattern in Niemann-Pick type C disease: a retrospective Swiss cohort study. Orphanet J Rare Dis. 2014;9:176.

48. Waterfang M, Chien YH, Imrie J, Rushton D, Schubiger D, Patterson MC. Dysphagia as a risk factor for mortality in Niemann-Pick disease type C: systematic literatura review and evidence from studies with miglustat. Orphanet J Rare Dis. 2012;7:76.

49. Colombia. Ministerio de Salud y Protección Social. Resolución 2048 de 2015. Bogotá: 2015.

50. Informes periódicos de Orphanet. Prevalencia de las enfermedades raras: Datos bibliográficos [Internet].2018;(2). [citado el 3 de abril de 2018]. Disponible en: http://www.orpha.net/ orphacom/cahiers/docs/ES/Prevalencia_de_las_enfermedades_raras_por_prevalencia_decreciente_o_casos.pdf

51. Colombia. Instituto Colombiano de Bienestar Familiar, Instituto Nacional de Salud, Profamilia. Encuesta Nacional de la Situación Nutricional en Colombia 2010. Bogotá: Ministerio de la Protección Social; 2011.

52. Aymé S, Kole A, Groft S. Empowerment of patients: lessons from the rare diseases community. Lancet. 2008;371(9629):2048-51. 